\title{
Salvage of a Floating Thrombosed Undersized Venous Stent by Crushing with New Stents
}

\author{
Taimur Saleem \\ The Rane Center for Venous and Lymphatic Diseases at St. Dominic's Hospital, Jackson, MS, USA
}

A 53-year-old male patient had symptoms of chronic venous insufficiency on the left leg, including previous venous ulceration (Clinical-Etiological-Anatomical-Pathophysiological classification (5), pain and swelling that caused limitations in his daily life despite the use of conservative measures. He had a history of left iliac venous stenting. Computed tomography venography scan showed a thrombosed 10-mm nitinol iliac venous stent that did not appear well apposed to the venous walls (Fig. 1). Furthermore, it showed an occluded stent with venous flow around it (Fig. 2A). A wire was maneuvered between the stent wall and the venous wall (Fig. 2B, C). The occluded nitinol stent was crushed by a large $(22 \mathrm{~mm} \times 40 \mathrm{~mm})$, high pressure balloon against the scaffold of the venous wall towards the left (Fig. 3). Next, two Wallstents (22 mm×70 mm each) and a Zstent ( $30 \mathrm{~mm} \times 50 \mathrm{~mm}$ ) were deployed (Fig. 4 and 5). The Zstent (Cook Medical, Bloomington, IN, USA) was used with the Wallstent (Boston Scientific, Marlborough, MA, USA) to provide additional radial force at the iliocaval confluence and to reduce the incidence of contralateral deep venous thrombosis [1-3]. Post-procedurally, the patient reported adequate symptom relief.

Accurate sizing of peripheral venous stents is critical for adequate decompression of peripheral venous hypertension. Undersized stents may embolize, migrate, or thrombose, as observed in this case. However, oversized stents may also
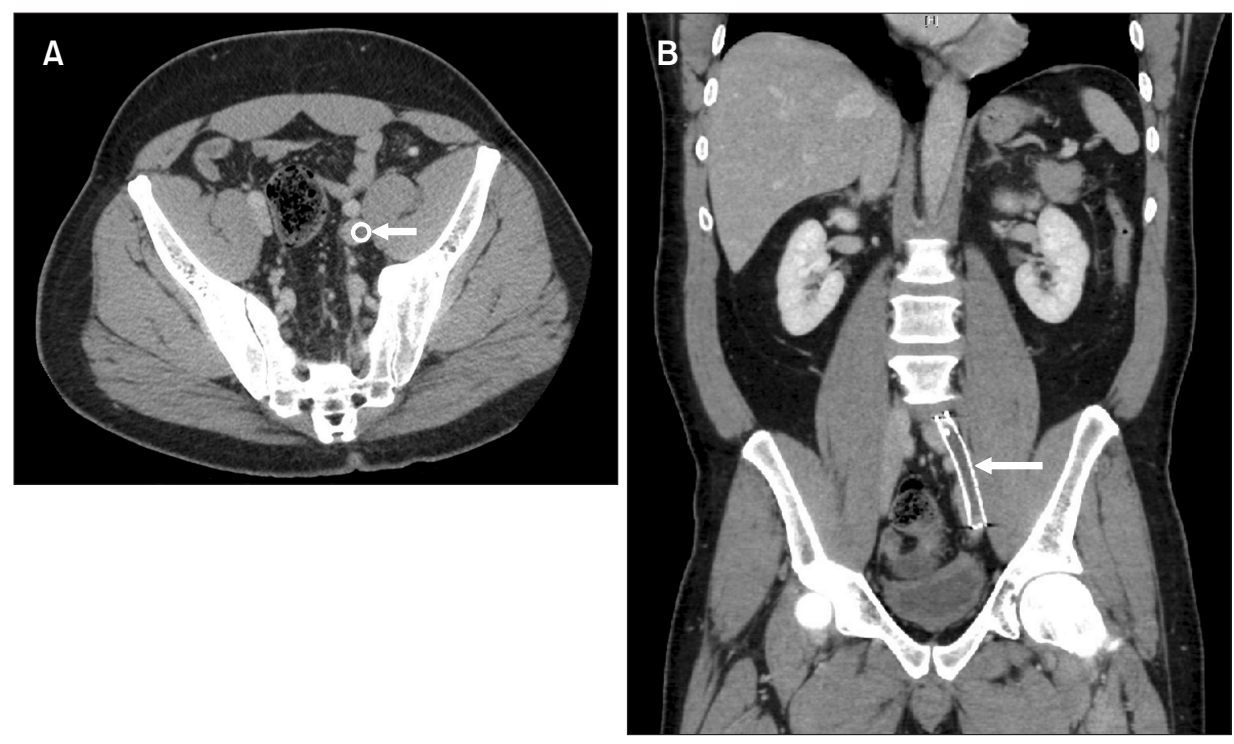

Fig. 1. Initial computed tomography images. (A) An axial view showed undersized iliac venous stent (arrow) with flow around it. (B) A coronal reformat showed a thrombosed iliac vein stent (arrow) that was not well apposed to the venous walls circumferentially.

Received July 16, 2021, Revised August 12, 2021, Accepted September 9, 2021, Published online September 28, 2021

Corresponding author: Taimur Saleem, The Rane Center for Venous and Lymphatic Diseases at St. Dominic's Hospital, 971 Lakeland Drive, Suite 401, Jackson, MS 39216, USA

Tel: 1-601-939-4230, Fax: 1-601-664-6694, Email: taimur@gmail.com, https://orcid.org/0000-0003-4467-3090

Copyright (C) 2021 The Korean Society for Vascular Surgery

This is an Open Access article distributed under the terms of the Creative Commons Attribution Non-Commercial License (http://creativecommons.org/licenses/by-nc/4.0) which permits unrestricted non-commercial use, distribution, and reproduction in any medium, provided the original work is properly cited.

Cite this article; Vasc Specialist Int 2021. https://doi.org/10.5758/vsi.210054 

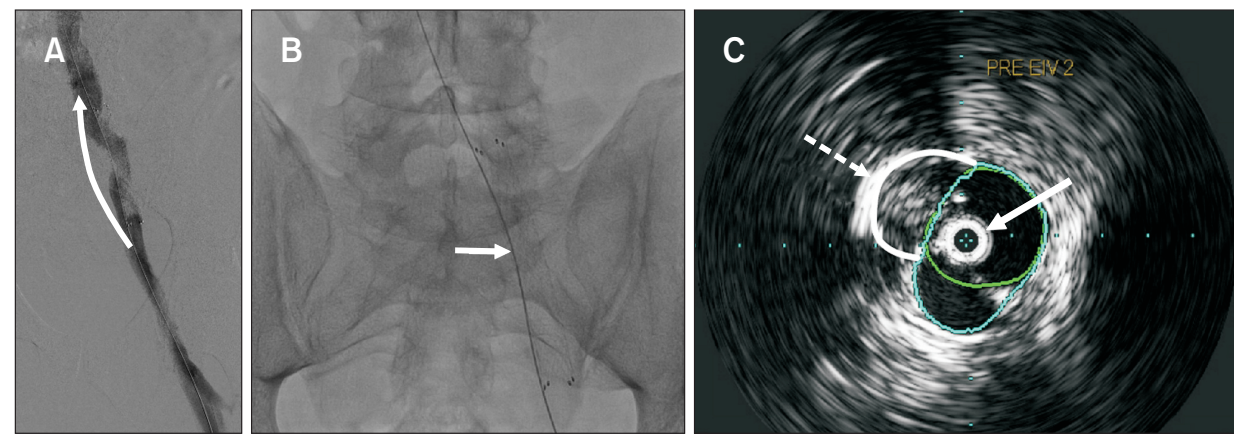

Fig. 2. (A) Venography showed a thrombus in stent and the venous flow around the "floating" stent (arrow). (B) Anteroposterior view showed a glide wire (arrow) between the venous wall and the stent wall. (C) Intravascular ultrasonography catheter located between the stent wall and the iliac vein wall in the center of the image (solid arrow). The dotted arrow indicates the location of the undersized stent which was not apposed to the vein wall circumferentially.

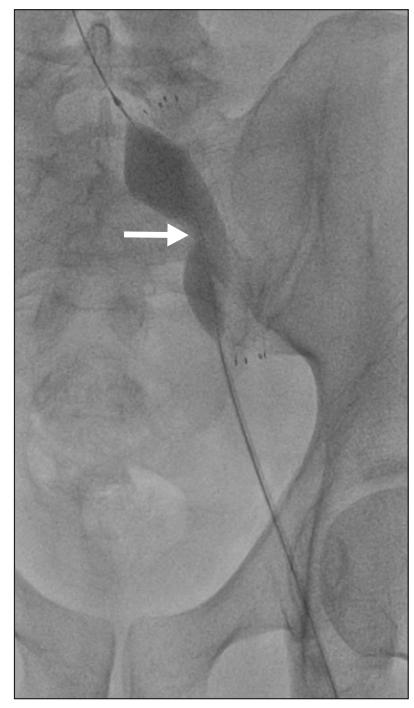

Fig. 3. Angioplasty balloon (arrow) was used to displace the previous nitinol stent to one side.

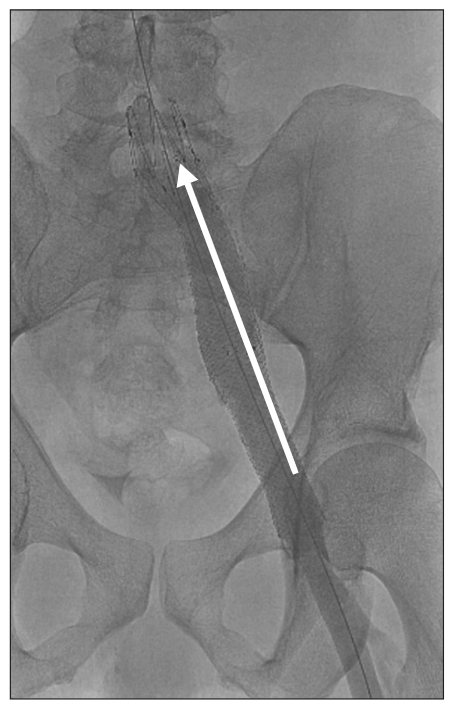

Fig. 4. Two Wallstents $(22 \mathrm{~mm} \times 70 \mathrm{~mm})$ and one Z-stent (30 $\mathrm{mm} \times 50 \mathrm{~mm}$ ) were deployed to restore complete luminal venous patency (arrow).


Fig. 5. Intravascular ultrasonography images after stenting. (A) Wallstent in the common femoral vein. (B) Wallstent in the external iliac vein. (C) Wallstent in common iliac vein. Note that the displaced previous stent can no longer be seen as a discrete circular ring. 
lead to occlusions and in-stent restenosis due to disturbances in shear stress, edge restenosis, and size mismatch between native and stented vein. Intravascular ultrasound is crucial in guiding iliac venous interventions and sizing of venous stents $[4,5]$.

\section{REFERENCES}

1) Jayaraj A, Noel C, Kuykendall R, Raju S. Long-term outcomes following use of a composite Wallstent-Z stent approach to iliofemoral venous stenting. J Vasc Surg Venous Lymphat Disord 2021;9:393-400.e2.

2) Murphy EH, Johns B, Varney E, Buck W, Jayaraj A, Raju S. Deep venous thrombosis associated with caval extension of iliac stents. J Vasc Surg Venous Lymphat Disord 2017;5:8-17.

3) Raju S, Ward M Jr, Kirk O. A modification of iliac vein stent technique. Ann Vasc Surg 2014;28:1485-1492.

4) Saleem T, Raju S. Comparison of intravascular ultrasound and multidimensional contrast imaging modalities for characterization of chronic occlusive iliofemoral venous disease: a systematic review. J Vasc Surg Venous Lymphat Disord 2021. doi: 10.1016/ j.jvsv.2021.03.022. [Epub ahead of print]

5) Saleem T, Knight A, Raju S. Diagnostic yield of intravascular ultrasound in patients with clinical signs and symptoms of lower extremity venous disease. J Vasc Surg Venous Lymphat Disord 2020;8:634-639. 